\title{
A Novel Role for the SMG-1 Kinase in Lifespan and Oxidative Stress Resistance in Caenorhabditis elegans
}

\author{
Ingrid Masse ${ }^{19 x}$, Laurent Molin ${ }^{19}$, Laurent Mouchiroud' ${ }^{1}$, Philippe Vanhems ${ }^{2}$, Francesca Palladino ${ }^{3}$, Marc \\ Billaud $^{1 *}$, Florence Solari ${ }^{1 *}$
}

1 Laboratoire de Génétique, Signalisation et Cancer, Université Claude Bernard Lyon 1, CNRS UMR5201 Domaine Rockefeller, Lyon, France, 2 Unité d'Epidémiologie et d'Hygiène Hospitalière, Hôpital Edouard Herriot, Hospices Civils de Lyon et Laboratoire d'Epidémiologie et de Santé Publique, Université de Lyon, Université Lyon 1, CNRS, UMR 5558, Laboratoire de Biométrie et Biologie Evolutive, Lyon, France, 3 Laboratoire de Biologie Moléculaire de la Cellule, Ecole Normale Supérieure de Lyon, CNRS UMR5161, Lyon, France

\begin{abstract}
The PTEN tumour suppressor encodes a phosphatase, and its daf-18 orthologue in Caenorhabditis elegans negatively regulates the insulin/IGF-1 DAF-2 receptor pathway that influences lifespan in worms and other species. In order to identify new DAF-18 regulated pathways involved in aging, we initiated a candidate RNAi feeding screen for clones that lengthen lifespan. Here, we report that smg-1 inactivation increases average lifespan in a daf-18 dependent manner. Genetic analysis is consistent with SMG-1 acting at least in part in parallel to the canonical DAF-2 receptor pathway, but converging on the transcription factor DAF-16/FOXO. SMG-1 is a serine-threonine kinase which plays a conserved role in nonsense-mediated mRNA decay (NMD) in worms and mammals. In addition, human SMG-1 has also been implicated in the p53-mediated response to genotoxic stress. The effect of smg-1 inactivation on lifespan appears to be unrelated to its NMD function, but requires the $p 53$ tumour suppressor orthologue cep-1. Furthermore, smg-1 inactivation confers a resistance to oxidative stress in a daf-18-, daf-16- and cep-1-dependent manner. We propose that the role of SMG-1 in lifespan regulation is at least partly dependent on its function in oxidative stress resistance. Taken together, our results unveil a novel role for SMG-1 in lifespan regulation.
\end{abstract}

Citation: Masse I, Molin L, Mouchiroud L, Vanhems P, Palladino F et al. (2008) A Novel Role for the SMG-1 Kinase in Lifespan and Oxidative Stress Resistance in Caenorhabditis elegans. PLoS ONE 3(10): e3354. doi:10.1371/journal.pone.0003354

Editor: Anne C. Hart, Massachusetts General Hospital/Harvard Medical School, United States of America

Received May 2, 2008; Accepted August 22, 2008; Published October 6, 2008

Copyright: (C) 2008 Masse et al. This is an open-access article distributed under the terms of the Creative Commons Attribution License, which permits unrestricted use, distribution, and reproduction in any medium, provided the original author and source are credited.

Funding: This study was supported by grants from CNRS and la Ligue Nationale Contre le Cancer. I.M. and L.M. were recipients of a fellowship from the Région Rhone-Alpes and from the French Ministry of Research, respectively. The funders had no role in study design, data collection and analysis, decision to publish, or preparation of the manuscript.

Competing Interests: The authors have declared that no competing interests exist.

* E-mail: Marc.Billaud@univ-lyon1.fr (MB); florence.solari@recherche.univ-lyon1.fr (FS)

a Current address: Institut de Génomique Fonctionelle de Lyon, Ecole Normale Supérieure de Lyon, INRA, Université Claude Bernard Lyon 1, CNRS UMR5242, Lyon, France

9 These authors contributed equally to this work.

\section{Introduction}

The insulin/IGF-1 DAF-2 receptor pathway is implicated in lifespan control in several species [1]. In C. elegans, DAF-2 signals to the PI3 kinase (PI3K) homolog AGE-1. AGE-1 generates phosphatidyl inositol 3, 4, 5 triphosphate, or PIP3, which is in turn responsible for the activation of the AKT-1, AKT-2 and SGK-1 serine threonine kinases in a PDK-1 dependent manner [2-5]. Phosphorylation of the FOXO transcription factor DAF-16 by these kinases inhibits DAF-16 function by inducing its sequestration into the cytoplasm [6-8]. The DAF-2 receptor pathway is downregulated by DAF-18, which is encoded by the C. elegans orthologue of the human tumor suppressor gene PTEN. Like PTEN, daf-18 encodes a PIP3 phosphatase that antagonizes the activity of AGE-1 in the DAF-2 pathway to regulate lifespan [913]. As daf-18 mutations suppress the lifespan phenotype of daf-2 and age-1 mutants, it has been proposed that the role of DAF-18 in lifespan regulation relies on inhibition of the insulin/IGF-1 signaling pathway (Mihaylova et al., 1999).

In order to identify new DAF-18 partners, we initiated a candidate RNAi feeding screen for clones that lengthen lifespan in a daf-18 dependent manner, focusing on potential protein kinases.
We identified one clone encoding the protein kinase SMG-1. Genetic analyses strongly suggest that SMG-1 acts in parallel to DAF-2, AGE-1 and AKT-1, but requires DAF-16, to modulate lifespan. SMG-1 is conserved across species and is involved in a mechanism responsible for the degradation of premature stop codon containing mRNA, also called NMD for "nonsense mediated mRNA decay" in C. elegans and in mammalian cells [14-16]. Interestingly the role of SMG-1 in lifespan appears to be unrelated to its function in NMD, but requires the $p 53$ C. elegans ortholog, cep-1, daf-18, and daf-16. Furthermore, our results uncover a role for SMG-1 in oxidative stress response that may be responsible for its effect on lifespan. Overall, our study unveils a novel role for SMG-1 in oxidative stress response and lifespan regulation that may be conserved in mammals.

\section{Results and Discussion}

smg-1 inhibition increases average lifespan in a daf-18 dependent manner

The screen was performed with $r f-3$ (pk1426) single and $r r f-3$ (pk1426); daf-18 (e1375) double mutants. The daf-18 (e1375) 
mutation is a hypomorphic allele of $d a f-18$, while the $r r f-3$ mutant was chosen because it shows an enhanced sensitivity to RNAi feeding [17]. As expected, RNAi of genes acting in the insulin pathway, including daf-2, age-1 and akt-1, resulted in a daf-18 dependent lengthening of lifespan, validating our experimental approach.

In addition, from the 269 hand picked clones tested (Table S1) we identified one corresponding to the $s m g-1$ gene. The average lifespan of the $r f-3$ strain was increased by $25 \%$ when worms were fed either one of the two non-overlapping RNAi clones for $s m g-1$ (C48B6.6 and C48B6.7) and the increase in lifespan was completely suppressed in $r r f-3(p k 1426)$; daf-18(e1375) mutants (Table 1; Figure 1A). Therefore, inhibition of $s m g-1$ by RNAi increases lifespan, and this effect requires DAF-18 activity.

We next tested whether the previously isolated $s m g-1$ (r861) null allele [18] also shows a lifespan phenotype. The average lifespan of smg-1(r861) mutant animals was not increased compared to wild- type animals (data not shown). Nonetheless, despite the fact that these mutants did not live longer than wild-type, we observed a delayed accumulation of the aging marker lipofuscine [19] during the first week of life of $s m g-1$ (r861) mutants, as observed in $s m g-1$ RNAi treated animals (data not shown).

Furthermore, in agreement with previously published data $s m g$ 1(r861) null mutants are associated with a fully penetrant protruding vulva phenotype $(94 \% ; n=205)$, while this phenotype was only observed in $31 \%(\mathrm{n}=969)$ of $s m g-1$ RNAi treated animals. The majority of other $s m g-1$ mutants we tested behave like the $s m g-1$ (r861) null allele (data not shown) besides $s m g-1$ (tm869) mutants (recently isolated by the Japanese C.elegans knockout consortium). An exception is the $s m g-1$ (tm869) allele, which results in a protruding vulva phenotype with similar penetrance $(47 \%$, $\mathrm{n}=351)$ to $s m g-1$ RNAi fed animals. Indeed, lifespan tests revealed that the $s m g-1$ (tm869) mutation increases average lifespan by more than 20\% (Figure 1B and Table 1).

Table 1. Effect of smg-1 inactivation on adult lifespan in different genetic backgrounds.

\begin{tabular}{|c|c|c|c|c|c|c|}
\hline Genotypes & RNAi & $\begin{array}{l}\text { Mean lifespan+/-SE } \\
\text { (days) }\end{array}$ & $\begin{array}{l}\text { Median } \\
\text { lifespan } \\
\text { (days) }\end{array}$ & $\begin{array}{l}P \text {-values } \\
\text { against } \\
\text { control }^{\text {a }}\end{array}$ & $\begin{array}{l}P \text {-values } \\
\text { against } \\
\text { specific group }\end{array}$ & $\begin{array}{l}\text { \# death/\# } \\
\text { total (\# trials) }\end{array}$ \\
\hline wild-type & & $16.2 \pm 0.4$ & 15 & & & 229/234(3) \\
\hline smg-1(tm869) & & $19.8 \pm 0.3$ & 21 & $<10^{-3}$ & & 187/351(3) \\
\hline \multirow[t]{22}{*}{$r r f-3(p k 1426)$} & control & $16.9 \pm 0.2$ & 15 & & & 701/925 (10) \\
\hline & smg-1 * & $21.2 \pm 0.3$ & 22 & $<10^{-3}$ & & $540 / 969(11)$ \\
\hline & smg-2 & $15.9 \pm 0.4$ & 15 & 0.002 & & 158/169 (2) \\
\hline & smg-4 & $18.9 \pm 0.6$ & 18 & 0.07 & & $94 / 179$ (2) \\
\hline & $s m g-5$ & $18.5 \pm 0.5$ & 18 & 0.074 & & 133/182(2) \\
\hline & smg-7 & $17 \pm 0.6$ & 15 & 0.9 & & 97/180 (2) \\
\hline & сер-1 & $18.6 \pm 0.5$ & 15 & 0.006 & & $246 / 300(3)$ \\
\hline & smg-1+cep-1 & $20 \pm 0.5$ & 19 & $<10^{-3}$ & $0.03^{b}$ & 227/294 (3) \\
\hline & daf-2 & $31.1 \pm 0.6$ & 32 & $<10^{-3}$ & & 289/351 (4) \\
\hline & age-1 & $23.6 \pm 0.5$ & 22 & $<10^{-3}$ & & 313/396 (4) \\
\hline & daf-2+age-1 & $30 \pm 0.8$ & 32 & $<10^{-3}$ & $0.48^{c}$ & $160 / 184(2)$ \\
\hline & smg-1+daf-2 & $37.3 \pm 0.7$ & 39 & $<10^{-3}$ & $<10^{-3 c} \mathrm{c}$ & 147/177 (2) \\
\hline & smg-1+age-1 & $31.1 \pm 0.8$ & 32 & $<10^{-3}$ & $<10^{-3 d}$ & 147/193 (2) \\
\hline & $a k t-1$ & $25.3 \pm 0.6$ & 26 & $<10^{-3}$ & & 138/198 (2) \\
\hline & smg-1+akt-1 & $29.7 \pm 0.6$ & 32 & $<10^{-3}$ & $<10^{-3 \mathrm{e}}$ & $134 / 196(2)$ \\
\hline & daf-18 & $14.7 \pm 0.3$ & 14 & $<10^{-3}$ & & $144 / 200(2)$ \\
\hline & daf-16 & $12.6 \pm 0.2$ & 12 & $<10^{-3}$ & & $156 / 289(3)$ \\
\hline & smg-1+daf-16 & $12.3 \pm 0.2$ & 12 & $<10^{-3}$ & $0.49^{f}$ & $114 / 199(2)$ \\
\hline & daf-19 & $20.9 \pm 0.6$ & 21 & $<10^{-3}$ & & 162/288 (2) \\
\hline & smg-1+daf-19 & $21.9 \pm 0.7$ & 21 & $<10^{-3}$ & $0.09^{b}$ & 101/174 (2) \\
\hline & daf-19+daf-18 & $14.4 \pm 0.3$ & 14 & $<10^{-3}$ & $0.52^{9}$ & $134 / 202(2)$ \\
\hline & daf- 19+daf-16 & $13.4 \pm 0.3$ & 12 & $<10^{-3}$ & $<10^{-3 h}$ & 105/198 (2) \\
\hline \multirow[t]{2}{*}{ daf-18(e1375); rrf-3(pk1426) } & control & $12.7 \pm 0.2$ & 12 & 0.79 & & 202/226 (3) \\
\hline & smg-1 & $12.5 \pm 0.3$ & 12 & & & 138/197 (3) \\
\hline \multirow[t]{2}{*}{$\operatorname{tax}-4(p 678) ; r r f-3(p k 1426)$} & control & $22.6 \pm 0.5$ & 24 & $<10^{-3}$ & & $146 / 201(2)$ \\
\hline & smg-1 & $26.5 \pm 0.6$ & 26 & & & 105/192 (2) \\
\hline
\end{tabular}

All experiments were carried out by RNAi feeding at $20^{\circ} \mathrm{C}$ (see experimental procedures). ${ }^{\text {a-i }}$ : $p$-values from a log rank test comparing RNAi treatment population to the vector

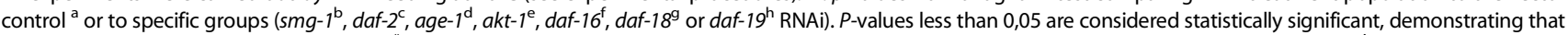
the two lifespan populations are different. ": results obtained with two independent RNAi feeding clones that gave similar results have been pooled.' The total number of individuals scored is shown followed by the number of individuals censored due to bursting vulva, bagging, or crawling off the agar. Data obtained in individual tests are reported in Table S2.

doi:10.1371/journal.pone.0003354.t001 
A

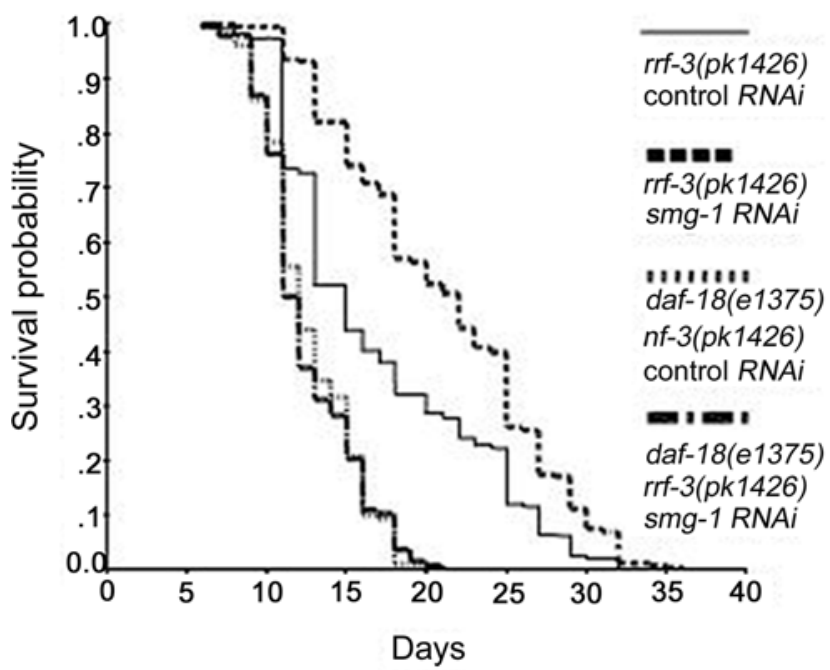

B

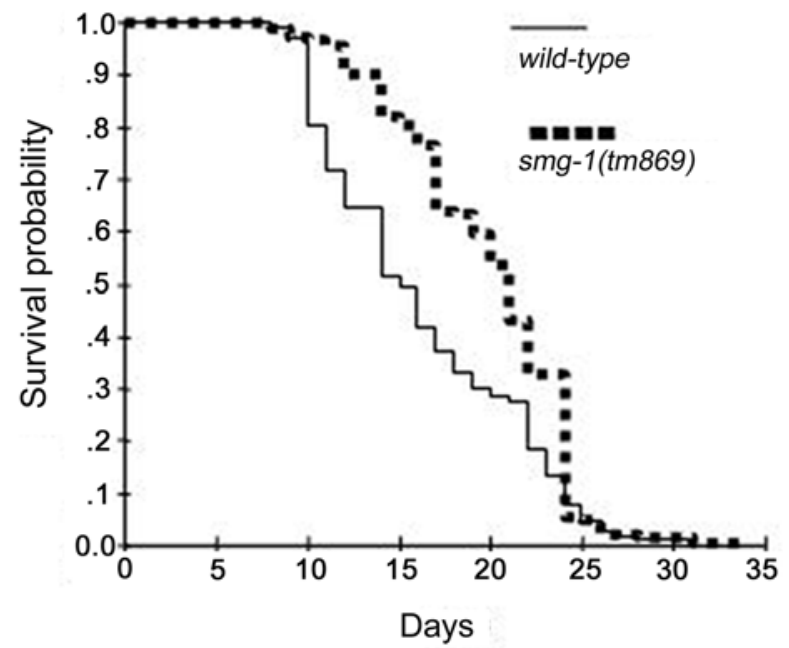

Figure 1. smg-1 inactivation increases mean lifespan in a daf-18 dependent manner. (A) Survival curves of $r r f-3(p k 1426)$ and daf-18(e1375); rrf-3(pk1426) mutants fed either bacteria not expressing any dsRNA (control RNAi) or bacteria expressing dsRNA that targets smg-1 (two independent clones gave similar results). For additional data, see Table 1. (B) Survival curves of $s m g-1$ (tm869) mutants and wild-type used for backcrosses (see Materials and Methods).

doi:10.1371/journal.pone.0003354.g001

Overall, our results suggest that RNAi mimics a hypomorphic mutation, while complete loss of SMG-1 function is deleterious and masks a longevity phenotype.

\section{SMG-1 may act in parallel of the insulin/IGF-1 DAF-2 receptor}

Since DAF-18 functions in the insulin/IGF-1 DAF-2 receptor pathway to regulate lifespan, we tested whether SMG-1 also acts in this signaling cascade.

We favored RNAi approaches to assess epistatic relationships between $s m g-1$ and the different components of the insulin pathway in order to analyse data in an isogenic background. RNAi of daf-2 increased lifespan by $84 \%$ compared to control RNAi (Table 1; Figure 2B). RNAi of both daf-2 and smg-1 further extended the average lifespan to 120\% (Table 1; Figure 2B). Similarly, the average lifespan of age-1 RNAi and akt-1 RNAi treated worms was further increased from 40 to $84 \%$ and from 50 to $75 \%$, respectively, when fed with $s m g-1$ RNAi (Table 1 ; Figure 2 $\mathrm{C}, \mathrm{D})$. Conversely, the lifespan of animals treated with RNAi for both daf-2 and age-1, which act in the same pathway, was not significantly different from the lifespan of daf-2 RNAi worms alone (Table 1; Figure 2A). Therefore, $s m g-1$ inactivation increases lifespan independently of daf-2, age-1 or akt-1. However, the extension of lifespan by $s m g-1$ RNAi was completely suppressed when daf-16 was inactivated by RNAi (Table 1; Figure 2E). Overall, these data suggest that SMG-1 may act in a pathway parallel to DAF-2, AGE-1 and AKT-1, but requiring DAF-16 activity.

Nonetheless, because gene inactivation by RNAi mimics a hypomorphic rather than a null mutation, we cannot formally exclude that the insulin receptor pathway partially contributes to the $s m g-1$ effect on lifespan.

DAF-16 function can be modulated through its nuclear localization $[6,7,20,21]$. To investigate whether SMG-1 controls DAF-16 sub-cellular localization, we made use of a strain carrying a daf-16::gfp reporter construct to visualize nuclear translocation in vivo [6]. DAF-16::GFP was localized in both the cytoplasm and the nucleus in all tissues of worms after $s m g-1$ inactivation by RNAi or by mutation (Figure 3), as observed in control worms. Conversely, under the same experimental conditions, daf-2 RNAi induced DAF-16::GFP nuclear accumulation (Figure 3). These results strongly suggest that SMG-1 does not regulate DAF-16 activity through its sequestration into the cytoplasm, and further support the idea that SMG-1 and DAF-2 may act in different pathways to regulate lifespan.

Nonetheless, these observations do not exclude that SMG-1 may behave as a weak enhancer of the insulin pathway, since Henderson and Johnson [6] showed that some age-1 mutants also fail to induce nuclear re-localization of this reporter. We thus addressed the relationship between the DAF-2 pathway and SMG1 by a third approach.

The DAF-2 pathway is also critical for controlling dauer formation. To further assess a potential functional link between SMG-1 and DAF-2 we tested the involvement of SMG-1 in dauer formation. One would predict that if the role of SMG-1 in lifespan control relies on its interaction with the DAF-2 pathway, then SMG-1 inhibition should increase dauer formation. However, we found that $s m g-1$ RNAi slightly increased the ability of worms to recover from larval arrest rather than enhancing dauer formation. When daf-2(e1370) or daf-2(e1370); rrf-3 (pk1426) double mutants were maintained at the semi-nonpermissive temperature $22^{\circ} \mathrm{C}$ [22], $78+/-7 \%$ of $s m g-1$ RNAi treated worms had reached the adult stage after 4 days, compared to $68+/-4.8 \%$ of worms grown on HT115 control bacteria $(P=0.02)$. These data strongly suggest that SMG-1 is not a broad positive regulator of the insulin pathway.

Overall our data support a model in which SMG-1 functions at least in part independently of DAF-2 to regulate lifespan.

The role of SMG-1 in lifespan control does not depend on its function in NMD

smg- 1 encodes a conserved serine threonine kinase involved in nonsense mediated mRNA decay (NMD), a mechanism responsible for the degradation of mRNAs containing a premature stop codon [14-16]. 


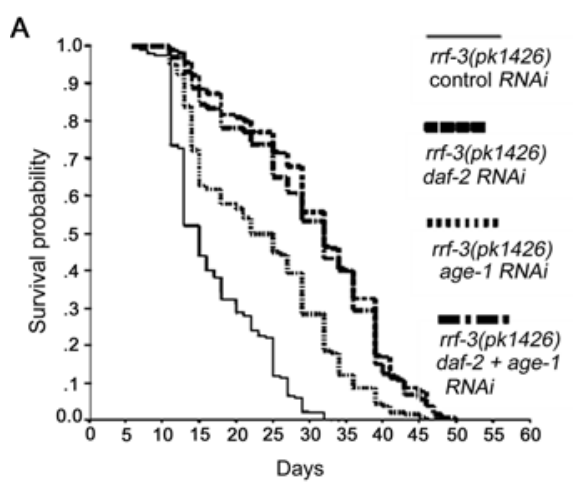

C
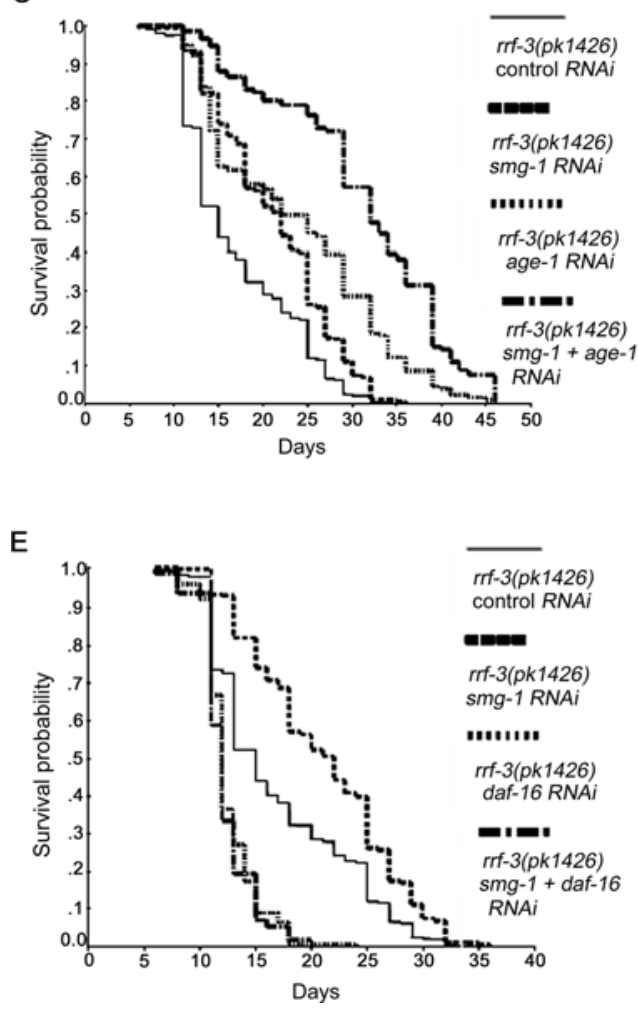
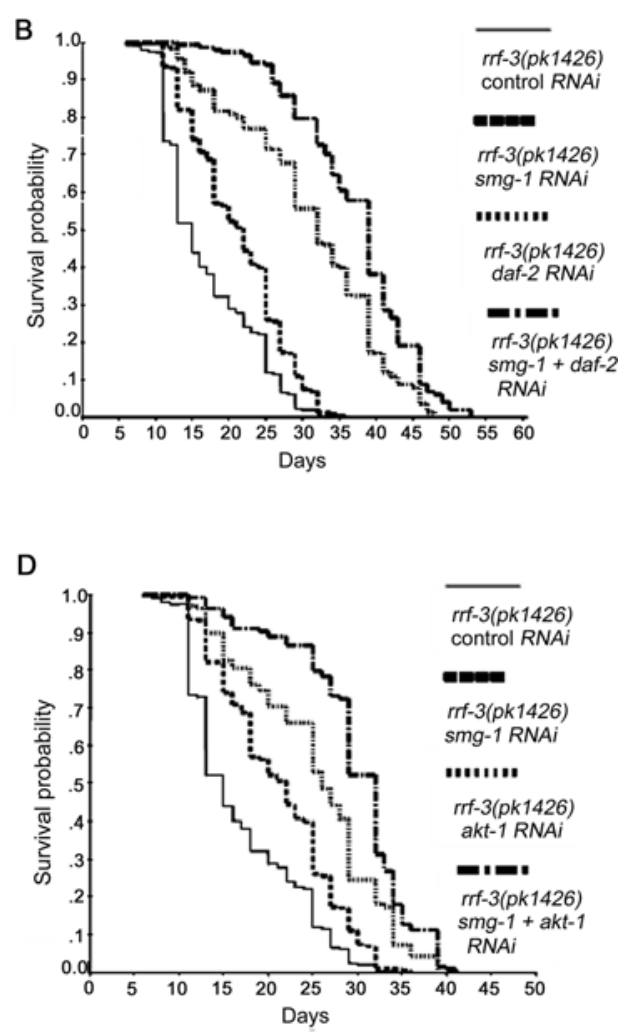

Figure 2. Interaction between $s m g-1$ and the insulin/IGF-1 signalling pathway genes for lifespan phenotype. (A) Survival curves of $r r f-$ 3(pk1426) mutants treated by daf-2 and/or age-1 RNAi. (B-E) Survival curves of rrf-3(pk1426) mutants treated by daf-2 (B), age-1 (C), akt-1 (D) and daf16 (E) RNAi alone or with $s m g-1$ RNAi. For additional data, see Table 1. doi:10.1371/journal.pone.0003354.g002

In addition to SMG-1, six evolutionary conserved SMG proteins are also required for NMD in C. elegans and in mammalian cells. Genetic studies have determined that smg genes are regulators of the phosphorylation state of SMG-2. SMG-1, SMG-3 and SMG-4 are required for the phosphorylation of SMG-2, whereas SMG-5, SMG-6 and SMG-7 are involved in its dephosporylation [23].

If the role of SMG-1 in lifespan relies on its function in NMD, inactivation of other $\mathrm{smg}$ genes should also have an effect on lifespan. The average lifespan of worms fed with $s m g-2, s m g-4, s m g-$ 5 or $s m g-7$ RNAi clones was not significantly increased (Table 1), suggesting that NMD inactivation may not be responsible for lifespan extension. To further explore this hypothesis, we assess NMD activity in $s m g-1$ RNAi treated worms. Longman et al. [24] developed an assay using transgenic strains expressing a GFP reporter constructs either with a natural stop codon or harboring a premature termination codon (PTC). Introduction of a PTC into the reporter induces a robust NMD response, as determined by the lack of GFP expression in transgenic worms. Conversely, smg-2 RNAi, which abrogates NMD, restores GFP expression [24]. Under our experimental conditions, GFP expression was induced in $100 \%$ of PTC transgenic worms fed with the $s m g-1$ RNAi clone (Figure 4), demonstrating the effectiveness of $s m g-1$ RNAi feeding in NMD inhibition. daf-18 and daf-16 are required for the $s m g-1$ dependent lifespan increase. We reasoned that if NMD inhibition is responsible for the role of $s m g-1$ in lifespan control, inactivation of daf-18 or daf-16 should antagonize this function and thus impede GFP expression in smg-1 RNAi treated PTC transgenic worms. Inactivation of either daf-18 or daf-16 by RNAi, which is sufficient to suppress the $s m g-1$ lifespan phenotype, did not reduce 

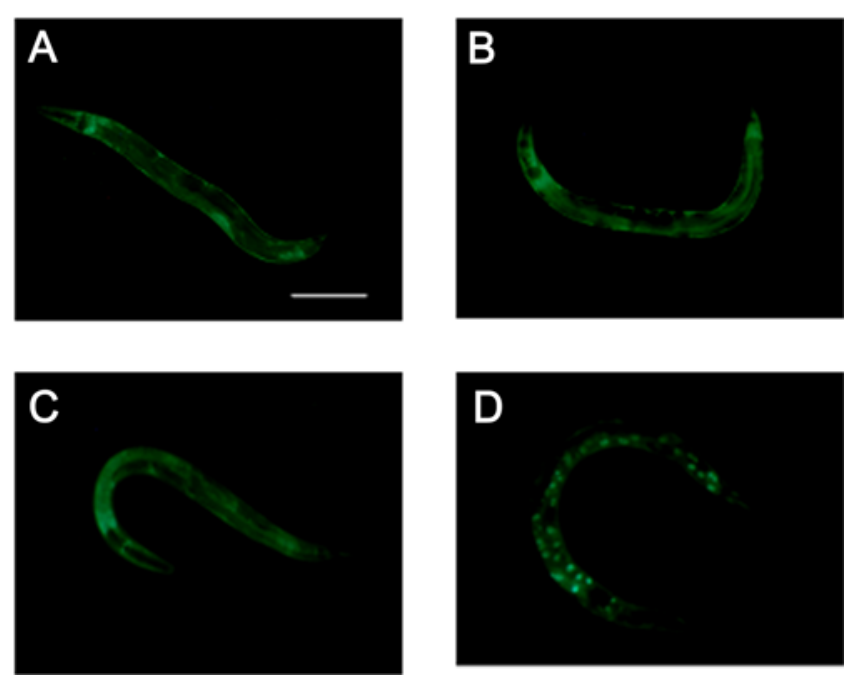

Figure 3. DAF-16::GFP sub-cellular localization is not altered by smg-1 inactivation. DAF-16::GFP is localized both in the cytoplasm and nuclei of all tissues at all developmental stages of $r r f-3$ (pk1426) (A) and wild-type (B) control animals as illustrated here for adults. Similar results were obtained for $r r f-3$ (pk1426) smg-1 RNAi-treated animals (C) and $s m g-1(r 861)$ single mutants (data not shown). (D) DAF-16::GFP accumulates in the nuclei of most cells in rrf-3(pk1426) animals treated with daf-2 RNAi. Scale bars: $100 \mu \mathrm{m}$. doi:10.1371/journal.pone.0003354.g003

the number of GFP expressing worms. Furthermore, the level of GFP expression in individual worms was unaffected or increased by $d a f-18$ and $d a f-16$ RNAi, respectively (Figure 4).

Overall our results show that there is no correlation between NMD inactivation and lifespan phenotypes, thus indicating that SMG-1 functions independently of NMD to regulate lifespan.

\section{p53/cep-1 is involved in smg-1 dependent lifespan modulation}

It was recently reported that human SMG-1 is functionally linked to the tumor suppressor checkpoint protein p53. hSMG-1 phosphorylates and stabilizes p53 in response to genotoxic stress induced by UV and $\gamma$ irradiation [25]. In worms, the $p 53$ homologue $c e p-1$ is required for DNA damage-induced apoptosis $[26,27]$. Interestingly, Arum et al. recently showed that cep-1 mutations also increase longevity without altering DAF-16::GFP nuclear localization [28]

We therefore tested whether $c$ ep- 1 is involved in the regulation of lifespan by $s m g-1$. cep- 1 RNAi partially suppressed the extension of lifespan due to $s m g-1$ inhibition (Table 1; Figure 5). The genetic interaction between cep- 1 and $s m g-1$ is specific, as cep- 1 RNAi alone did not reduce lifespan (Table 1; Figure 5). These results indicate that when $s m g-1$ is inactivated, cep- 1 is required to extend lifespan.

smg-1 inactivation confers resistance to oxidative stress

Resistance to oxidative stress is a hallmark of many longevity mutants in C. elegans [29]. Consistently, we observed that worms were more resistant to oxidative stress induced by paraquat when $s m g-1$ was inactivated by RNAi or by mutation, as $47 \%$ of worms were still alive after 7 days of paraquat treatment compared to $7 \%$ for control RNAi (Figure 6 A, B). Conversely, daf-18 and daf-16 RNAi inhibited the resistance of worms to oxidative stress compared to control RNAi and dramatically reduced the stress resistance induced by $s m g-1$ inactivation (Figure $6 \mathrm{~A}, \mathrm{~B}$ ). These
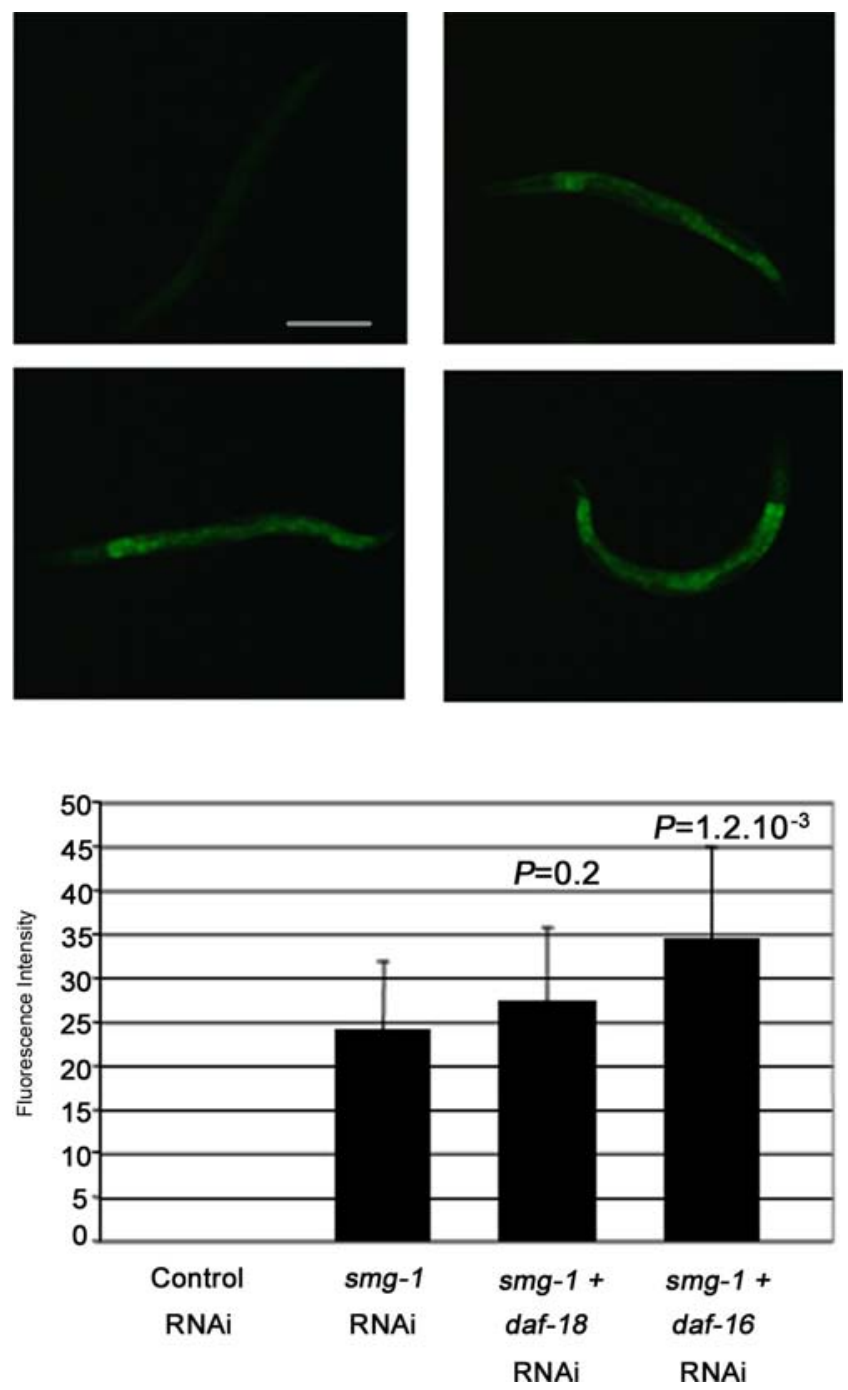

Figure 4. The SMG-1 dependent effect on NMD regulation is not suppressed upon DAF-18 or DAF-16 inactivation. Animals carrying the PTC transgenic reporter were fed with the different RNAi clones and scored for GFP expression (see experimental procedures). Three independent experiments were carried out and gave similar results. (A) Animals treated with the control $(n=80)$ lack GFP expression. Conversely, $100 \%$ of worms treated by smg-1 RNAi $(n=80)(B)$, or by $s m g-1$ and daf-18 RNAi $(n=60)(C)$, or by $s m g-1$ and daf-16 RNAi $(n=50)(D)$ showed GFP expression. Scale bar: $200 \mu$ m. (E) GFP quantification using arbitrary units (see experimental procedure). The error bars reflect the variation between animals. $P$ values correspond to comparison of average GFP intensity between the test group and worms treated with smg-1 RNAi using Student's $t$ test. doi:10.1371/journal.pone.0003354.g004

results show that SMG-1 requires DAF-16 and DAF-18 to confer oxidative stress resistance as well as to function in lifespan control. A correlation between lifespan and oxidative stress resistance phenotypes was also observed for the genetic interaction between $d a f-2$ and $s m g-1$, as the resistance to oxidative stress of $d a f-2$ RNAi treated animals was further increased by $s m g-1$ inactivation (Figure $6 \mathrm{~A}, \mathrm{~B} ; \mathrm{p}<10^{-3}$ ).

To further explore the relationship between lifespan and resistance to oxidative stress phenotypes, we investigated the role of $c e p-1 / p 53$ in oxidative stress resistance. Under experimental conditions where cep-1 RNAi increased lifespan (Figure 5), resistance to oxidative stress of RNAi treated animals increased 


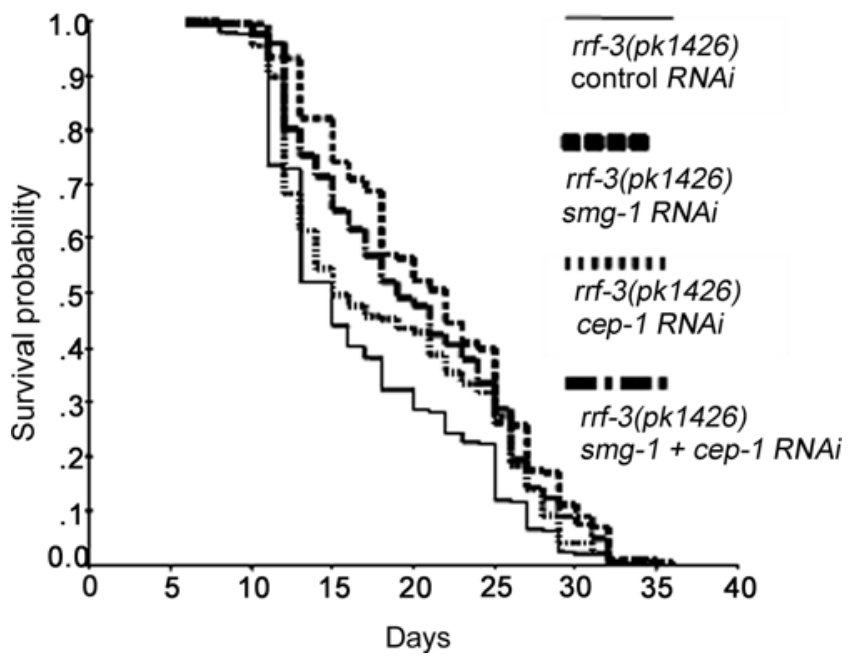

Figure 5. SMG-1 requires CEP-1 to regulate lifespan. Survival curves of rrf-3(pk1426) mutants treated by smg-1 and/or cep-1 RNAi. This figure uses the same data set as Table 1. doi:10.1371/journal.pone.0003354.g005 significantly compared to control animals (Figure $6 \mathrm{C}$, D; $\left.\mathrm{p}<10^{-3}\right)$. Conversely, the stress resistance of $s m g-1$ RNAi animals was reduced by cep-1 RNAi (Figure $6 \mathrm{C}, \mathrm{D} ; \mathrm{p}<10^{-3}$ ), as expected from the partial suppression observed for the lifespan phenotype (Figure 6).

Altogether, our results show that CEP-1 has opposing effects on oxidative stress resistance depending on the presence or absence of $s m g-1$. This apparent paradox may be explained by the role of p53 in mammalian cells, where it displays either pro-oxidant or antioxidant functions depending on the level of oxidative stress (high or low, respectively, [30]. In wild-type animals treated with paraquat, where the level of oxidative stress is high, p53 plays a pro oxidant function, thus explaining the beneficial effect of reducing its expression. Upon $s m g-1$ inactivation, the levels of oxidative stress may be lower; p53 could play an antioxidant function in this context [30], explaining the deleterious effect of its removal.

Overall, our results show that positive and negative regulators of SMG-1 activity in lifespan regulation act in a similar manner in the oxidative stress response. These data support the hypothesis that the resistance to oxidative stress of $s m g-1$ animals may be responsible for
A

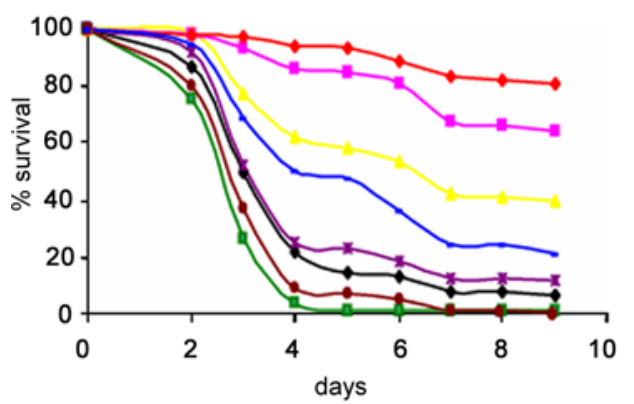

B

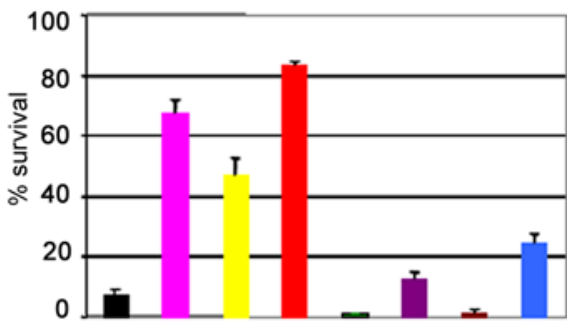

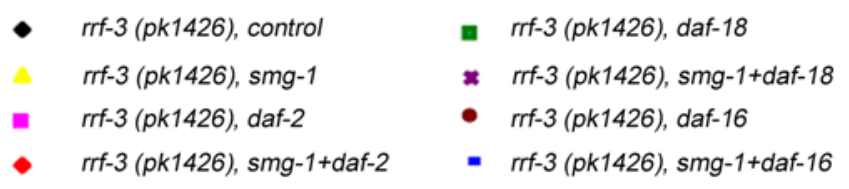

C

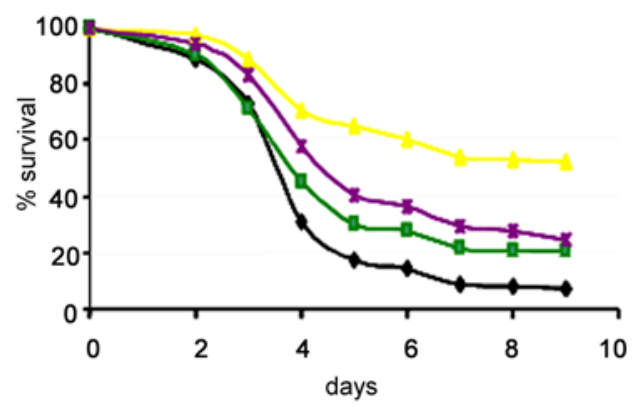

D

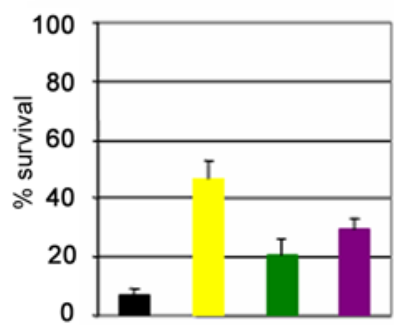

- rrf-3 (pk1426), control rrf-3 (pk1426), smg-1
- $\quad r f-3(p k 1426), c e p-1$

* $\quad r f-3$ (pk1426), smg-1+ep-1

Figure 6. SMG-1 regulates resistance to oxidative stress in a DAF-18, DAF-16 and CEP-1 dependent manner. (A, C) Survival curves of RNAi treated animals in presence of paraquat (see materials and methods). (B,D) Histograms presented as mean \pm SEM at day 7 of paraquat treatment. $s m g-1$ (r861) null mutants behave similarly to $s m g-1$ RNAi animals (data not shown). Genetic interactions for oxidative stress resistance between smg-1 and either daf-2, daf-18 or daf-16 (A, B) and between smg-1 and cep-1 (C, D). doi:10.1371/journal.pone.0003354.g006 
A

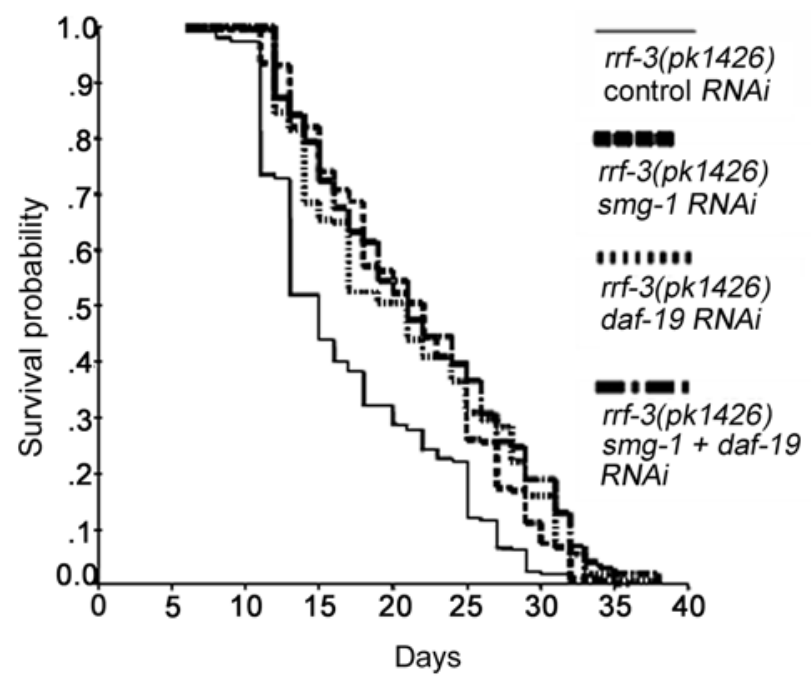

C

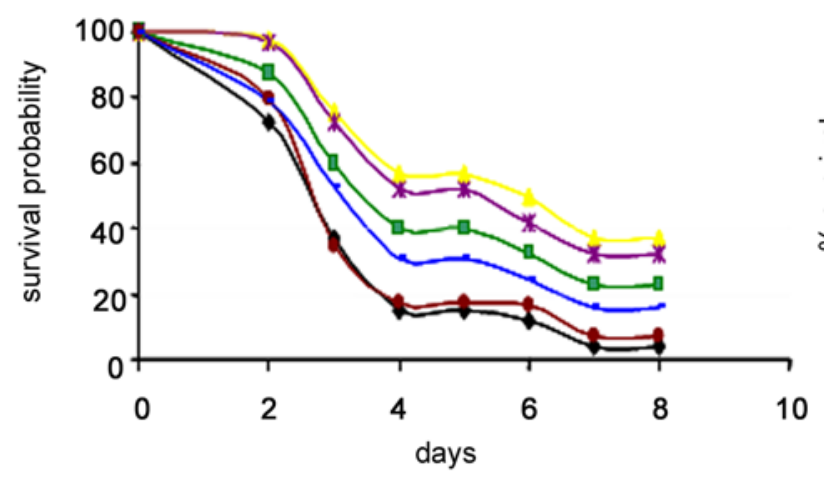

B

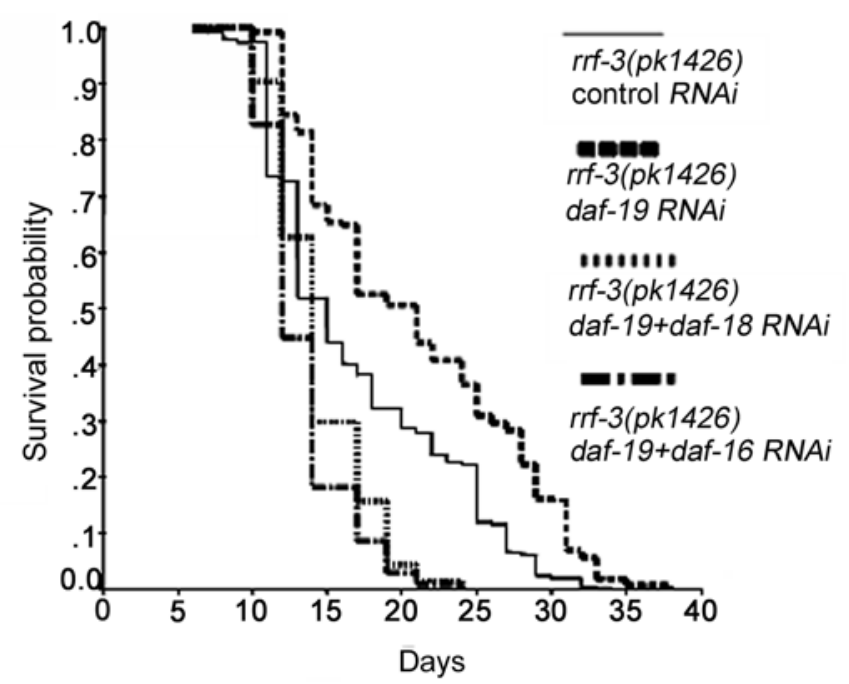

D

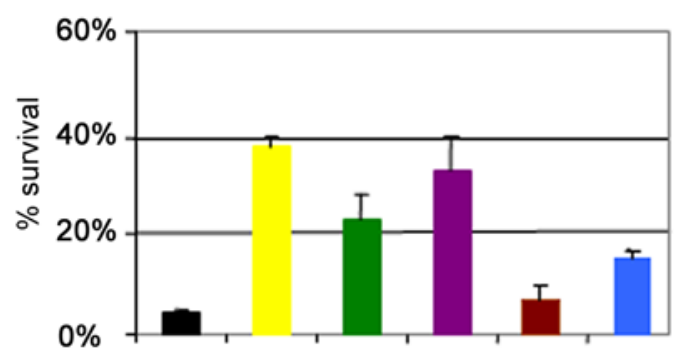

- $\quad r r f-3(p k 1426) ;$ control rrf-3(pk1426); smg-1

rrf-3(pk1426); daf-19
× $\quad r f-3(p k 1426) ; s m g-1+d a f-19$

- rrf-3(pk1426); daf-19+daf-18

- rrf-3(pk1426); daf-19+daf-16

Figure 7. Genetic interaction between $s m g-1$ and daf-19 for lifespan and oxidative stress resistance phenotypes. (A, B): Survival curves of $r f-3(p k 1426)$ mutants treated with $s m g-1$ and/or daf-19 RNAi (A) and treated with daf-19 RNAi alone or in combination with daf-18 or daf-16 RNAi (B). Curves are drawn from data set of Table 1. (C) Survival curves of RNAi treated animals in presence of paraquat (see Materials and Methods). (D) Histograms presented as mean \pm SEM at day 7 of paraquat treatment. Genetic interactions for oxidative stress resistance between daf- 19 and either smg-1, daf-18 or daf-16 are shown. doi:10.1371/journal.pone.0003354.g007

their increased lifespan phenotype. However, other mechanisms may also be involved since $d a f-16$ (or $d a f-18$ ) inactivation is sufficient to suppress $s m g-1$ lifespan regulation without completely inhibiting the $s m g-1$ stress resistance phenotype.

A role for SMG-1 in sensory neuron signaling for lifespan regulation

Several observations prompted us to investigate the role of $s m g-1$ in the regulation of lifespan via sensory neurons. Firstly, among the different mechanisms that modulate $C$. elegans lifespan, mutations in sensory neurons lengthen lifespan in a daf-16 dependent manner [31]. Secondly, a number of genes that act in the nervous system have been shown to be refractory to RNAi in a wild type context, but efficiently inactivated in an $r r f-3$ RNAi hyper-sensitive background [17]. Similarly, smg- 1 RNAi increased lifespan in RNAi hyper-sensitive backgrounds such as $r r f-3$ and $p p w-1$ [32], but not in wild-type animals (Figure 1 and data not shown).

We therefore asked whether $s m g-1$ genetically interacts with tax4 and daf-19, two genes involved in lifespan regulation by sensory neurons [31]. The lifespan of $\operatorname{tax}-4(p 678) ; \operatorname{rff}-3(p k 1426)$ double mutants was increased when these animals were fed with $s m g-1$ RNAi (Table 1), suggesting that tax-4 and smg-1 act independently to regulate lifespan. Conversely, the lifespan of animals fed with both daf-19 and smg-1 RNAi was not significantly lengthened compared to either RNAi alone (Table 1; Figure 7A). Furthermore, similarly to $s m g-1$, the lifespan increase caused by daf-19 RNAi inactivation was fully suppressed by daf-16 RNAi (Table 1; Figure 7B), as previously reported for daf-16(mu86); daf-19(m86) 
double mutants [31], as well as by daf-18 RNAi (Table 1; Figure $7 \mathrm{~B})$.

daf-19, which encodes an evolutionary conserved RFX-type transcription factor, is a master gene for the development of a ciliary module in C. elegans [33]. In order to assess the role of SMG-1 in sensory neuron formation, animals were stained with $\mathrm{DiO}$, a fluorescent probe which enters through functional cilia (see material and methods).We counted on average $10.2+/-0.2$ $(\mathrm{n}=50) ; 9.4+/-0.3(\mathrm{n}=42) ; 9.6+/-0.2(\mathrm{n}=29)$ and $0(\mathrm{n}=21)$ ciliated neurons, respectively, in $r f-3$ animals maintained on control bacteria, $r r f-3$ animals on daf-19 RNAi, in daf-12(sa204) mutants, and in daf-19(m86); daf-12(sa204) mutants. Therefore, while daf-19(m86) mutants do not stain at all [33], inhibition of daf19 expression by RNAi is not strong enough to interfere with sensory neurons development, as also observed by others (Peter Swoboda, personal communication). Furthermore, smg-1 RNAi treated animals stained a similar number of sensory neurons as animals fed on control bacteria $(10.5+/-0.2, n=40)$. Overall our results show that $5 m g-1$ inhibition by RNAi does not compromise sensory neurons formation. These results do not however exclude a possible function for SMG-1 in sensory perception. The $s m g-1$ predicted promoter contains the canonical DAF-19 target sequence [34], suggesting that $s m g-1$ expression may be regulated by DAF-19. As daf-19 expression is restricted to sensory neurons, smg-1 may well play a role in these cells to regulate lifespan. Nonetheless, the SMG-1 mode of action differs from the previously described pathways, as sensory mutants, but not $s m g-1$ inactivation, affect DAF-16 nuclear translocation [31].

As observed upon $s m g-1$ inactivation, resistance to oxidative stress was increased after daf-19 RNAi, and was suppressed when daf-19 was inactivated in combination with daf-16 or daf-18 (Figure 7C, D). Furthermore, in contrast to daf-2 and $s m g-1$ double RNAi, inactivation of daf-19 did not confer higher resistance to $s m g-1$ RNAi animals. These results strongly suggest that SMG-1 may function with DAF-19 to regulate both lifespan and oxidative stress.

In conclusion, we identified $s m g-1$ as a novel gene involved in lifespan regulation. Furthermore, our results suggest that SMG-1 may act independently of DAF-2 and requires DAF-18/PTEN, DAF-16/FOXO and CEP-1/p53 to regulate lifespan.

In mammalian cells, p53 and FOXO3A can act as cofactors to regulate transcription [35]. Furthermore, PTEN has also been shown to interact with and to stabilize the p53 protein [36]. Thus, it is tempting to speculate that SMG-1 may affect DAF-16 transcriptional activity via the regulation of PTEN and p53. Interestingly, the physical interaction between p53 and PTEN has recently been shown to be regulated by oxidative stress [37], and their functional crosstalk does not require the lipid phosphatase activity of PTEN [37]. This is in agreement with our results strongly suggesting that SMG-1 acts independently of the PI 3 kinase AGE-1. Finally, pull down experiments revealed that PTEN and hSMG-1 physically interact in human cells [38]. It is therefore possible that mammalian ortholog of $s m g-1$ control lifespan and the response to oxidative stress in mammals. Recent data suggests a function for SMG-1 both in the oxidative stress response [39] and a role in apoptosis unrelated to the suppression of nonsense-mediated mRNA decay [40]. Understanding the molecular interactions and mechanisms of the pathway involving SMG-1 in aging will be the challenge for future studies.

\section{Materials and Methods}

\section{Strains}

Strains used were as follows: wild-type strains N2 Bristol, daf18(e1375) IV [13,41], rrf-3(pk1426) II [17], tax-4(p678) III [42], daf-19(m86) II; daf-12(sa204) X and daf-12(sa204) [33]. smg1(r861) I [18] and smg-1(tm869), obtained from the C.elegans knockout consortium directed by Pr Mitani, were outcrossed three additional times. The PTCxi strain [24] was kindly provided by D. Longman (J.F. Caceres Lab, MRC, Edinburgh, Scotland).

To construct double mutants daf-18 (e1375) IV; rrf-3 (pk1426) II, $r r f$-3(pk1426) males were crossed to daf-18(e1375) hermaphrodites and $\mathrm{F} 2$ progeny were assayed for sterility at $25^{\circ} \mathrm{C}$ and incapacity to form dauer on overgrown plates. Double mutants $\operatorname{tax}-4(p 678)$ III; $r f-3$ (pk1426) II were generated by crossing tax-4(p678) III hermaphrodites to $r r-3(p k 1426)$ II males. F2 progeny were screened for sterility at $25^{\circ} \mathrm{C}$ and dauer constitutive formation at $27^{\circ} \mathrm{C}$. To obtain single mutants unc-54(r293) I, N2 males were mated to $s m g-3(r 930) ~ I V$; unc-54(r293) $I$ and paralyzed F2 were isolated. To generate $r f-3(p k 1426)$ mutants carrying the DAF16::GFP transgene, $r f-3(p k 1426)$ males were crossed to TJ356 [6] hermaphrodites. $\mathrm{F} 2$ rollers were assayed for sterility at $25^{\circ} \mathrm{C}$.

\section{RNAi experiments}

Bacterial feeding RNAi experiments were carried out essentially as described previously [43]. Briefly, single colonies of HT115 bacteria containing plasmids of interest were first grown overnight in LB with $100 \mu \mathrm{g} / \mathrm{ml}$ ampicillin and $12.5 \mu \mathrm{g} / \mathrm{ml}$ tetracyclin and then for $8 \mathrm{~h}$ in LB with $100 \mu \mathrm{g} / \mathrm{ml}$ ampicillin. Bacteria were seeded directly onto NGM plates containing $2 \mathrm{mM}$ IPTG and $25 \mu \mathrm{g} / \mathrm{ml}$ carbenicillin. Clones used: C48B6.6 and C48B6.7 (smg-1), F46B6.3 (smg-4), W02D3.8 (smg-5), Y43B6A.a (smg-7), B0334.8 (age-1), C12D8.10 (akt-1), R13H8.1 (daf-16), F52B5.4 (cep-1), F33H1.1 (daf-19) and T07A9.6 (daf-18) have been purchased from. daf-2 and $s m g-2$ clones were kindly provided by C. Kenyon lab (University of California, San Francisco, USA) and D. Longman (J.F. Caceres Lab, MRC, Edinburgh, Scotland) respectively. Each clone has been sequenced to confirm its identity.

Double RNAi experiments were carried out by mixing the bacterial cultures directly before seeding the NGM plates. Controls were RNAi clone 50\% diluted with vector control RNAi bacteria.

\section{Lifespan assays}

Animals were grown on regular NGM plates at $20^{\circ} \mathrm{C}$ until reaching the $\mathrm{L} 4$ stage and then transferred to RNAi plates $(\mathrm{F} 0)$. F0 adults were removed after $24 \mathrm{~h}$ and F1 L4 were transferred to $10 \mu \mathrm{M}$ 5-fluorodeoxyuracile (5-FU, Sigma-Aldrich, Steinheim, Germany) containing plates to prevent growth of progeny. Lifespan assays were performed at $20^{\circ} \mathrm{C}$. The day of the shift is counted as day 0 in the adult lifespan assay. Control and experimental animals were transferred in parallel to fresh RNAi plates once a week. Lifespan was assessed every 2-3 days and animals were scored as dead when they ceased moving and responding to prodding. Animals that crawled off the plate, had a "protruding vulva" or an "exploded vulva" phenotype were censored. smg-1 RNAi was also performed in absence of 5-FU and gave similar results (data not shown).

Survival analyses were performed using the Kaplan Meier method and the significance of differences between survival curves calculated using the log rank test. The statistical software used was SPSS, Version 11.5 (SPSS, Chicago, IL, USA) and all Pvalues $<0.05$ were considered significant.

\section{Assessment of NMD activity in living worms}

Animals carrying the PTC transgenic reporter [24] were fed at $20^{\circ} \mathrm{C}$ with the control clone only or with the smg- 1 RNAi clone mixed either with the control, or daf-18, or daf-16 RNAi clones. F1 animals were scored for GFP expression at the L4 stage. For GFP 
intensity quantification, animals were photographed under a GFP filter and the average brightness was determined for each photograph by Lucia Nikon software. All images were handled identically. At least 30 animals per RNAi conditions were averaged.

\section{Stress resistance assays}

Synchronously cultured animals were kept on NGM plates at $20^{\circ} \mathrm{C}$ until the young adult stage. For each strain, $5 \times 20$ young adults were transferred on Paraquat (methylviologene, SigmaAldrich, Steinheim, Germany) containing plates (90 $\mu \mathrm{l}$ of $150 \mathrm{mM}$ Paraquat added on top of NGM plates already seeded with HT1 15 bacteria). Surviving animals were scored every day during 8-9 days. At least three independent RNAi experiments have been conducted for each clone tested. $P$-values were calculated using the t-Student test to determine differences in oxidative stress resistance.

\section{Larval arrest assays}

Five young adults daf-2(e1370) ; rrf-3(pk1426) double mutants were fed at $22^{\circ} \mathrm{C}$ with either HT1 15 or $s m g-1$ RNAi bacteria, then removed 24 hours later. $\mathrm{F} 1$ progenies were followed every day and the numbers of worms that have reached the adult stage were counted at day 4. Numbers are given for 3 independent experiments.

\section{Observation of DAF-16::GFP sub-cellular localization and DiO staining}

The sub-cellular localization of the DAF-16::GFP protein was analyzed in the $s m g-1$ (r861) mutant background and after $s m g-1$ RNAi inactivation in a $r r f-3(p k 1426)$ mutant background by fluorescence microscopy under a GFP filter. About 10 worms were

\section{References}

1. Bonafe M, Barbieri M, Marchegiani F, Olivieri F, Ragno E, et al. (2003) Polymorphic variants of insulin-like growth factor I (IGF-I) receptor and phosphoinositide 3-kinase genes affect IGF-I plasma levels and human longevity: cues for an evolutionarily conserved mechanism of life span control. J Clin Endocrinol Metab 88: 3299-3304.

2. Dorman JB, Albinder B, Shroyer T, Kenyon C (1995) The age-1 and daf-2 genes function in a common pathway to control the lifespan of Caenorhabditis elegans. Genetics 141: 1399-1406.

3. Hertweck M, Gobel C, Baumeister R (2004) C. elegans SGK-1 is the critical component in the Akt/PKB kinase complex to control stress response and life span. Dev Cell 6: 577-588.

4. Morris JZ, Tissenbaum HA, Ruvkun G (1996) A phosphatidylinositol-3-OH kinase family member regulating longevity and diapause in Caenorhabditis elegans. Nature 382: 536-539.

5. Paradis S, Ruvkun G (1998) Caenorhabditis elegans Akt/PKB transduces insulin receptor-like signals from AGE-1 PI3 kinase to the DAF-16 transcription factor. Genes Dev 12: 2488-2498.

6. Henderson ST, Johnson TE (2001) daf-16 integrates developmental and environmental inputs to mediate aging in the nematode Caenorhabditis elegans. Curr Biol 11: 1975-1980.

7. Lee RY, Hench J, Ruvkun G (2001) Regulation of C. elegans DAF-16 and its human ortholog FKHRL1 by the daf-2 insulin-like signaling pathway. Curr Biol 11: $1950-1957$.

8. Lin K, Hsin H, Libina N, Kenyon C (2001) Regulation of the Caenorhabditis elegans longevity protein DAF-16 by insulin/IGF-1 and germline signaling. Nat Genet 28: 139-145.

9. Gil EB, Malone Link E, Liu LX, Johnson CD, Lees JA (1999) Regulation of the insulin-like developmental pathway of Caenorhabditis elegans by a homolog of the PTEN tumor suppressor gene. Proc Natl Acad Sci U S A 96: 2925-2930.

10. Mihaylova VT, Borland CZ, Manjarrez L, Stern MJ, Sun H (1999) The PTEN tumor suppressor homolog in Caenorhabditis elegans regulates longevity and dauer formation in an insulin receptor-like signaling pathway. Proc Natl Acad Sci U S A 96: 7427-7432.

11. Ogg S, Ruvkun G (1998) The C. elegans PTEN homolog, DAF-18, acts in the insulin receptor-like metabolic signaling pathway. Mol Cell 2: 887-893.

12. Rouault JP, Kuwabara PE, Sinilnikova OM, Duret L, Thierry-Mieg D, et al. (1999) Regulation of dauer larva development in Caenorhabditis elegans by daf18, a homologue of the tumour suppressor PTEN. Curr Biol 9: 329-332. mounted on agar pads ( $2 \%$ agarose with $5 \mathrm{mM}$ tetramisole) to avoid DAF-16::GFP translocation due to stress [8]. At least 20 animals were examined for each developmental stage (embryo, L1, L2, L3, L4 and adult).

Sensory neurons were stained by incubating L4 worms in M9 containing DiO (Molecular Probes) at $10 \mu \mathrm{g} / \mathrm{ml}$ final concentration for two hours. Worms were then transfered to plates for one hour and observed by fluorescence microscopy under a GFP filter.

\section{Supporting Information}

Table S1 List of clones tested in the screen.

Found at: doi:10.1371/journal.pone.0003354.s001 (0.03 MB XLS)

Table S2 Lifespan data for individual experiments Found at: doi:10.1371/journal.pone.0003354.s002 (0.33 MB DOC)

\section{Acknowledgments}

All strains except smg-1(tm869) were provided by the Caenorhabditis Genetics Center, which is funded by the NIH National Center for Research Resources (NCRR). We thank the C.elegans knockout consortium directed by Pr Mitani for $s m g-1$ (tm869) mutants and C. Kenyon for the kind gift of the daf-2 clone. We thank Jeremy for his help in handling figures. We are very grateful to $\mathrm{D}$. Longman and $\mathrm{X}$. Caceres for communicating results and providing reagents before publication.

\section{Author Contributions}

Conceived and designed the experiments: FS. Performed the experiments: IM LM LM FS. Analyzed the data: IM FS. Contributed reagents/ materials/analysis tools: IM PV FS. Wrote the paper: FP FS. Financial support: MB.

13. Solari F, Bourbon-Piffaut A, Masse I, Payrastre B, Chan AM, et al. (2005) The human tumour suppressor PTEN regulates longevity and dauer formation in Caenorhabditis elegans. Oncogene 24: 20-27.

14. Denning G, Jamieson L, Maquat LE, Thompson EA, Fields AP (2001) Cloning of a novel phosphatidylinositol kinase-related kinase: characterization of the human SMG-1 RNA surveillance protein. J Biol Chem 276: 2270922714 .

15. Grimson A, O'Connor S, Newman CL, Anderson P (2004) SMG-1 is a phosphatidylinositol kinase-related protein kinase required for nonsensemediated mRNA Decay in Caenorhabditis elegans. Mol Cell Biol 24: 7483-7490.

16. Yamashita A, Ohnishi T, Kashima I, Taya Y, Ohno S (2001) Human SMG-1, a novel phosphatidylinositol 3-kinase-related protein kinase, associates with components of the mRNA surveillance complex and is involved in the regulation of nonsense-mediated mRNA decay. Genes Dev 15: 22152228.

17. Simmer F, Moorman C, van der Linden AM, Kuijk E, van den Berghe PV, et al (2003) Genome-wide RNAi of C. elegans using the hypersensitive rrf-3 strain reveals novel gene functions. PLoS Biol 1: E12.

18. Hodgkin J, Papp A, Pulak R, Ambros V, Anderson P (1989) A new kind of informational suppression in the nematode Caenorhabditis elegans. Genetics 123: 301-313.

19. Garigan D, Hsu AL, Fraser AG, Kamath RS, Ahringer J, et al. (2002) Genetic analysis of tissue aging in Caenorhabditis elegans: a role for heat-shock factor and bacterial proliferation. Genetics 161: 1101-1112.

20. Lin K, Dorman JB, Rodan A, Kenyon C (1997) daf-16: An HNF-3/forkhead family member that can function to double the life-span of Caenorhabditis elegans. Science 278: 1319-1322.

21. Oh SW, Mukhopadhyay A, Svrzikapa N, Jiang F, Davis RJ, et al. (2005) JNK regulates lifespan in Caenorhabditis elegans by modulating nuclear translocation of forkhead transcription factor/DAF-16. Proc Natl Acad Sci U S A 102: 4494-4499.

22. Lee SS, Lee RY, Fraser AG, Kamath RS, Ahringer J, et al. (2003) A systematic RNAi screen identifies a critical role for mitochondria in C. elegans longevity. Nat Genet 33: 40-48.

23. Anders KR, Grimson A, Anderson P (2003) SMG-5, required for C.elegans nonsense-mediated mRNA decay, associates with SMG-2 and protein phosphatase 2A. Embo J 22: 641-650. 
24. Longman D, Plasterk RH, Johnstone IL, Caceres JF (2007) Mechanistic insights and identification of two novel factors in the $\mathrm{C}$. elegans NMD pathway. Genes Dev 21: 1075-1085.

25. Brumbaugh KM, Otterness DM, Geisen C, Oliveira V, Brognard J, et al. (2004) The mRNA surveillance protein hSMG-1 functions in genotoxic stress response pathways in mammalian cells. Mol Cell 14: 585-598.

26. Derry WB, Putzke AP, Rothman JH (2001) Caenorhabditis elegans p53: role in apoptosis, meiosis, and stress resistance. Science 294: 591-595.

27. Schumacher B, Hofmann K, Boulton S, Gartner A (2001) The C. elegans homolog of the p53 tumor suppressor is required for DNA damage-induced apoptosis. Curr Biol 11: 1722-1727.

28. Arum O, Johnson TE (2007) Reduced expression of the Caenorhabditis elegans p53 ortholog cep-1 results in increased longevity. J Gerontol A Biol Sci Med Sci 62: 951-959.

29. Finkel T, Holbrook NJ (2000) Oxidants, oxidative stress and the biology of ageing. Nature 408: 239-247.

30. Sablina AA, Budanov AV, Ilyinskaya GV, Agapova LS, Kravchenko JE, et al. (2005) The antioxidant function of the p53 tumor suppressor. Nat Med 11: 1306-1313.

31. Apfeld J, Kenyon C (1999) Regulation of lifespan by sensory perception in Caenorhabditis elegans. Nature 402: 804-809.

32. Tijsterman M, Okihara KL, Thijssen K, Plasterk RH (2002) PPW-1, a PAZ/ PIWI protein required for efficient germline RNAi, is defective in a natural isolate of C. elegans. Curr Biol 12: 1535-1540.

33. Swoboda P, Adler HT, Thomas JH (2000) The RFX-type transcription factor DAF-19 regulates sensory neuron cilium formation in C. elegans. Mol Cell 5: $411-421$.
34. Efimenko E, Bubb K, Mak HY, Holzman T, Leroux MR, et al. (2005) Analysis of xbx genes in C. elegans. Development 132: 1923-1934.

35. Nemoto S, Finkel T (2002) Redox regulation of forkhead proteins through a p66shc-dependent signaling pathway. Science 295: 2450-2452.

36. Li AG, Piluso LG, Cai X, Wei G, Sellers WR, et al. (2006) Mechanistic insights into maintenance of high p53 acetylation by PTEN. Mol Cell 23: 575-587.

37. Chang CJ, Mulholland DJ, Valamehr B, Mosessian S, Sellers WR, et al. (2008) PTEN nuclear localization is regulated by oxidative stress and mediates p53dependent tumor suppression. Mol Cell Biol 28: 3281-3289.

38. Crockett DK, Fillmore GC, Elenitoba-Johnson KS, Lim MS (2005) Analysis of phosphatase and tensin homolog tumor suppressor interacting proteins by in vitro and in silico proteomics. Proteomics 5: 1250-1262.

39. Gehen SC, Staversky RJ, Bambara RA, Keng PC, O'Reilly MA (2008) hSMG-1 and ATM sequentially and independently regulate the $\mathrm{G}(1)$ checkpoint during oxidative stress. Oncogene.

40. Oliveira V, Romanow WJ, Geisen C, Otterness DM, Mercurio F, et al. (2008) A protective role for the human SMG-1 kinase against tumor necrosis factoralpha-induced apoptosis. J Biol Chem 283: 13174-13184.

41. Riddle DL, Swanson MM, Albert PS (1981) Interacting genes in nematode dauer larva formation. Nature 290: 668-671.

42. Dusenbery DB, Sheridan RE, Russell RL (1975) Chemotaxis-defective mutants of the nematode Caenorhabditis elegans. Genetics 80: 297-309.

43. Kamath RS, Martinez-Campos M, Zipperlen P, Fraser AG, Ahringer J (2001) Effectiveness of specific RNA-mediated interference through ingested doublestranded RNA in Caenorhabditis elegans. Genome Biol 2: RESEARCH0002. 